\title{
Tracce di donne nel primo Trecento
}

Abstracts: Il saggio passa in rassegna alcune "tracce" di volgare femminile nell'Italia del primo Trecento, in Veneto e in Toscana; si sofferma in particolare sulle condizioni di produzione e trasmissione delle poche testimonianze identificabili e sulla precarietà documentale del "segno" qualificabile al femminile (serie rimanti, scritture avventizie o sopravvivenze orali).

The paper scans some "tracks" of female vernacular texts recorded in Italy (Veneto and Tuscany) during the first half of the fourteenth century. It especially observes the conditions of production and transmission of surviving female writings and underlines, from a documental point of view, the contingent character of the "female sign" (rhymes, later glosses and additions, oral witnesses).

Parole chiave: Female Textuality, Women readers/writers, Manuscript Tradition, Religious Literature

Supporre un'autrice, una scrivente, e comunque un'origine femminile, dentro un testo volgare comporta spesso l'analisi di un dislivello - almeno fino alla seconda metà del secolo XIV, ovvero prima che si delineassero in modo sempre più netto i profili biografici e culturali delle più antiche scrittrici d'Europa (fatte salve alcune precoci apparizioni). Soprattutto se documentaria, la scrittura delle donne, e sul piano materiale e su quello testuale e linguistico, la si insegue di norma nella diafasia, nelle "irregolarità", ovvero misurandone gli scarti rispetto al canone della tradizione maschile - scrittoria e paleografica, da un lato, sintattica, morfologica e lessicale, dall'altro. La convergenza di questi caratteri diasistemici consente talvolta di sostanziare l'ipotesi su un'autorialità femminile, di rilevare appena una fisionomia linguistica e culturale, per contrasto a ciò che appare norma e maschile. Altre volte ad importare è il contenuto enunciativo del testo femminile, o presunto tale; la Compiuta Donzella del Canzoniere Vaticano, ad esempio, se da un lato condivide la cultura di base, tipologica, del lirismo maschile e romanzo - «però non mi ralegra fior né foglia», ${ }^{1}$

1 Poeti del Duecento, I, p. 434.

Lorenzo Mainini, Sapienza Università di Roma

Ә Open Access. ( 2021 Lorenzo Mainini, published by De Gruyter. (c) BY This work is licensed under the Creative Commons Attribution 4.0 International License. 
«non cre que m'esiauza / per fueill ni per flor», ${ }^{2}$ a mero titolo d'esempio -, e dunque il suo retroterra non parrebbe distinguerla dagli uomini con cui rima, introduce tuttavia nei suoi versi quei riferimenti extra-testuali, quei realia, alla vita delle donne che fanno dei suoi sonetti un "testo al femminile", dove s'intenda con l'espressione “al femminile” quell'armamentario, anch'esso tipologico, dal quale non solo le donne, ma anche gli uomini (nei contrasti ad esempio), possono ricavare i tratti di un'enunciazione di genere, e dunque ugualmente autentici o simulati: il padre che la destina in sposa contro il suo volere - «ca lo mio padre m'ha messa 'n errore, / (. . .) / donar mi vole a mia forza segnore»; ${ }^{3}$ il preferire il chiostro al matrimonio obbligato - «lo padre mio mi fa stare pensosa / ca di servire a Cristo mi distorna» ${ }^{4}$-, e via dicendo.

In ogni caso il dislivello che contraddistingue la testualità femminile, alle origini delle letterature volgari, non è solo diafasico, pertinente cioè alle variazioni contestuali dell'atto enunciativo; la capacità che si attribuisce, o si attribuiva, al testo femminile di richiamare in superficie dimensioni storicamente sommerse dell'enunciazione è pure diacronico; in tal senso il testo femminile, o il "testo al femminile", per le sue specifiche rispetto ai canoni della tradizione prevalente, lo si spiegava talvolta, almeno fino a un secolo fa, come il relitto di tempi remoti: è femminile, è diverso e dunque è arcaico. In estrema sintesi è questo il sillogismo che ha organizzato alcuni approcci ai testi delle chansons de toile o alle danze francesi della reverdie. ${ }^{5}$ Per la Francia si potrebbe aggiungere: testo femminile, e dunque rurale e popolare oltre che semplicemente arcaico. E lo stesso approccio è valso, secondo variabili geografiche e culturali, per le preghiere d'amore e i lamenti in voce di donna nelle kharjas iberiche, arabo-romanze, o in Italia per certi componimenti spesso in forma "zagialesca" e di ballata. In definitiva la concomitanza di cultura extra-cortese, popolare e popolareggiante, di forme metriche e tematiche primordiali, tutto ciò unito ad un io poetico che parla al femminile, ha favorito il prodursi d'un magazzino della memoria romanza - guardato ormai da tempo con debite precauzioni rispetto alla critica precedente - al cui interno si sarebbero ritrovate le origini

2 Dansas provenzali del secolo XIII, p. 95.

3 Poeti del Duecento, I, p. 434.

4 Poeti del Duecento, I, p. 435.

5 Si pensi, fra tutti, alla storiografia di Jeanroy: «toute cette poésie, comme le montrent ses rythmes, très appropriés à régler le chant et la danse, dut être à l'origine composée de chansons à danser. Cette circonstance explique que le type le plus ancien soit la chanson de femme ou de jeune fille. (. . .). Il est donc à penser que c'étaient les jeunes filles qui composaient à leur usage des chansons où elles avaient d'abord peint leurs propres sentiments (. . .), et c'est ainsi à des femmes qu'il faudrait faire remonter les plus anciens essais lyriques en pays roman», Jeanroy, Les origines de la poésie, pp. 444-445. 
letterarie e le donne, al posto della stanza di canzone i ritornelli dei canti comuni, invece delle corti i prati di maggio. Questo magazzino della memoria, prodotto di tanta filologia ottocentesca, e messo tra parantesi dalla critica moderna, è in definitiva il frutto dei dislivelli caratteristici di molta antica testualità al femminile: scarti diafasici, quanto alle condizioni linguistiche e stilistiche, e diacronici, quanto a i tempi.

Del resto è all'insegna del dislivello che gli stessi letterati volgari concepiscono non solo il parlare delle donne, ma anche il parlare alle donne. Oltre il rinvio d'obbligo a Dante, alla Vita nova e alla donna «malagevole d'intendere li versi latini» $(\mathrm{XXV}, 4),{ }^{6}$ e perciò causa movente della prima poesia in volgare, lo stesso concetto, lo stesso dislivello e la conseguente necessità di colmarlo, era già nella piena tradizione cortese: «chançon legiere a entendre», canzone facile da capire e da ascoltare, è quella che Conon de Béthune invia non solo a «chascuns», ma in particolare «a la millor», alla donna: la légèreté della canzone, la sua dichiarata semplicità, risponde al bisogno d'abolire un'eventuale mediazione («autres messaigiers») tra l'enunciatore maschile e la donna destinataria. ${ }^{7}$

La ricerca d'una testualità femminile negli spazi aperti dal dislivello trova ugualmente ricadute d'ambito materiale, grafico. Si prenda ad esempio il Pontificale di Sens (cfr. Fig. 11.1), relato dal codice parigino lat. 934, primo-duecentesco: le carte iniziali sono occupate dai giuramenti di fedeltà al proprio vescovo prestati dagli abbati e dalle badesse della diocesi francese. Nella serie omologata delle elaborate scritture documentarie, opera verosimile d'uno scriptor professionista, compare il giuramento d'una certa badessa Aupes; la difformità grafica di quest'ultimo giuramento, che spezza la serie delle scritture documentarie, per introdurre invece una textualis semplice e per certi versi insicura, consente d'ipotizzare - come in effetti è avvenuto - l'intervento autografo della monaca, che di propria mano, senza temere il risultato minore rispetto alla circostante norma dello scriptor curiale, verga il suo personale giuramento. Ecco quindi l'ipotesi d'una scrittura femminile ricavata di nuovo per contrasto, misurandone il dislivello nel diasistema delle scritture interne al documento.

Dalla scrittura alla lingua, il dislivello è nuovamente in gioco nei processi di mediazione, ovvero lungo la trasmissione scritta del discorso femminile. All'inizio del Trecento, in Italia il caso più celebre è quello d'Angela da Foligno e del suo Liber: dislivello, nella misura in cui la lingua e l'enunciazione - volgare - della donna si vogliono mediate dal resoconto latino del frate scrittore,

6 Dante Alighieri, Vita nuova - Rime, pp. 210-211.

7 Les chansons de Conon de Béthune, p. 1. 
che converte in discorso l'esperienza, oralmente riferita, della mistica. Da qui il dislivello fra la reale enunciazione della donna e la testualità che materialmente ne è il vettore. ${ }^{8}$ Ulteriore riverbero della natura scalare che fra Due e Trecento denota la voce femminile è la sua rappresentazione letteraria. Al riguardo l'analisi può sfumarsi: se in effetti nel perimetro di certa letteratura, per lo più cortese, l'immagine d'una donna in relazione col libro e con la scrittura (a dire il vero, il più delle volte, la lettura) non è rara, ${ }^{9}$ in altri contesti della produzione letteraria il dislivello di cui trattiamo emerge più chiaramente. Istruttivo è un passaggio della Vita agiografica d'Umiltà da Faenza, la donna romagnola che lungo la seconda metà del secolo XIII - fino agl'inizi del XIV -, fra la città d'origine e Firenze, diviene un modello di vita claustrale e religiosità femminile. ${ }^{10}$

E stando in tal modo rinchiusa, le altre suore forse per sollazzo, con ciò fosse cosa ch'ella [Umiltà] non fosse in leggere molto amaestrata, un dì la chiamarono (. . .), sì.lle commisono che legesse alla seconda mensa, secondo l'usanza delle monache. Allora la religiosissima e umile donna (. . .) prese il libro (. . .), l'aperse e cominciò a dire: "Non vogliate dispregiare le opere di Dio, però ch'elle sono tutte giuste e veraci”; sopra la qual parola senpre cogl'occhi levata al cielo disse tanto altissime cose, che tutto il convento commosse a correre e a vedere e udire tanto maravigliosa e disusata cosa. E quando le fu fatto segno che ponesse fine alla sua letione, e dicendo quella parola che s'usa dire, cioè: $T u$ autem, domine, miserere nobis, (. . .), amaestrata dal santo spirito, ottimamente fece fine. E le cose le quali ella lesse giamai niuno, né prima né poi, poté nel predetto libro trovare.

Sembra che il racconto metta in gioco, sotto le spoglie del discorso ispirato, il tema dell'oralità, quale obbligo per la comunicazione non-alfabetizzata della donna e in modi, per certi versi, identici-opposti all'oralità che caratterizza il Liber di Angela da Foligno. Se in quest'ultima la voce della donna, attraverso il resoconto scritto del frate, si codifica in lettera e in libro, nel caso d'Umiltà invece l'oralità, la voce femminile, si produce attraverso la de-codifica della lettera scritta e del libro: quest'ultimo è obliterato, trasceso, dal discorso orale della santa. Nel libro dal quale Umiltà dovrebbe leggere non si ritrovano le parole dette dalla donna; di nuovo - per quanto in gioco vi sia l'esibizione d'un miracolo - si vuole aprire un dislivello, un'asimmetria, fra il testo e l'enunciazione femminile.

Quanto fin qui detto rappresenta una serie, episodica, di possibili focalizzazioni sul tema della donna e della testualità, latina e volgare, in epoca duetrecentesca, ovvero piuttosto alta, almeno per quel che attiene alla questione

8 Si veda Vanelli Coralli, Mistica e nuovo concetto, pp. 453-484.

9 Si veda Mancini, Lettori e lettrici di romanzi, pp. 155-176.

10 Le Vite di Umiltà da Faenza; la citazione che segue alle pp. 31-32. 
sotto esame; in effetti la cronologia non è indifferente. Se si percorre la bibliografia sul tema si vedrà come le fonti cronologicamente risalenti scarseggino e come, di conseguenza, una storia - che sia filologica, linguistica e paleografica del volgare femminile di norma si risolva, alle sue origini, in una storia tardotrecentesca e soprattutto quattrocentesca. Gli scavi sull'autografia condotti da Luisa Miglio, ad esempio, e i documenti da lei pubblicati risalgono largamente al secolo XV ${ }^{11}$ E così molti lavori sulle scritture private, gli epistolari femminili e la loro lingua. Le cause sono ovvie: la documentazione manca, o scarseggia - fra le recenti eccezioni, alcune carte documentarie, due-trecentesche, di donne veneziane, discusse da Formentin; ${ }^{12}$ questa scarsità attiene ovviamente alla posizione della donna nella società basso-medievale, i canali precari della sua educazione, l'alfabetizzazione saltuaria e spesso domestica, il perimetro circoscritto della sua enunciazione - la vita famigliare e la religiosità; tutti aspetti che incidono grandemente sulla possibilità di registrare e tramandare un testo femminile e che, ancora nel Quattrocento, fissano i limiti di ciò che le donne consegnano allo scritto. Si tratta per lo più di lettere ai figli, mariti e parenti, che esprimono gli affetti e l'incombenza domestica, talvolta la cura d'affari economici, laddove a scrivere sia una laica; oppure minime comunicazioni ufficiali, tra una monaca e un prelato o il protettore d'un monastero, se a scrivere sono le religiose. In tutti questi casi si rileva lo sforzo da parte della donna d'agire in un campo dell'esistenza quello della scrittura manu propria - che mobilita complessivamente la sua personalità: la lingua, la fonetica e la morfologia, i suoi nessi sintattici, fin lì orali, devono farsi segni grafici. Di qui anche le ritrosie personali, oltre che storiche; com'è quella, documentata, d'una Lena Strozzi, e discussa da Miglio, ${ }^{13}$ in una lettera al marito: «io ti scrivo il meglo che so, sì che non ti far beffe di me». Esiste poi l'universo delle donne copiste; ma anche qui la maggior parte delle testimonianze - salvo i casi ridotti, e in latino, provenienti da scriptoria altomedievali si colloca nello stesso avanzato arco cronologico. ${ }^{14}$

Se queste sono le precondizioni della ricerca, valide fino al secolo $\mathrm{XV}$, si può dedurre quali siano le difficoltà nel trattare la questione per l'arco cronologico più antico, due-trecentesco, ed inoltre, come vuole il Convegno, per testi letterari, più che documentari, e nella prospettiva della diglossia latino-volgare; e in Italia, per giunta, dove sono più rare quelle occasioni d'alta committenza femminile, che invece talvolta si hanno in Francia, intorno alle corti - da quella antica di Champagne sino a quella regale di Bianca di Castiglia, coi volgarizzamenti che pare

11 Miglio, Governare l'alfabeto.

12 Formentin, Scritture femminili.

13 Miglio, Governare l'alfabeto, p. 287.

14 Si vedano i manoscritti schedati nel repertorio online Donne e cultura scritta nel Medioevo. 
possano ricondursi al suo patronato. Per la letteratura italiana due-trecentesca, nella sua articolazione latino-volgare, la posizione delle donne è dunque difficile da ricostruire su basi testuali e linguistiche. Se mettiamo da parte quelle figure dubbie o del tutto false della tradizione poetica, o ancora le canzoni di donna e di lontananza, esperimenti maschili in voce di donna, davvero mancano testi sufficientemente eloquenti. Quanto segue è quindi l'individuazione, per frammenti, d'alcune tracce, con lo scopo d'evidenziare la precarietà di quelle spie documentali che nei testi permettono di supporre la sottostante presenza di una donna.

Iniziamo fuori dalla Toscana; da alcune preghiere in versi, in volgare veronese, note agli studi sin dal 1864 coi Monumenti antichi di dialetti italiani d'Adolfo Mussafia ${ }^{15}$ e più recentemente tornate all'attenzione di Zeno Verlato ${ }^{16}$ e Alina Zvonareva, ${ }^{17}$ che in misura differente si sono occupati dei testi e dei codici relatori. Le preghiere che ci interessano sono tramandate all'interno di sillogi devote e agiografiche, settentrionali, d'origine almeno in parte veronese e poi stratificate nel lombardo-veneto, insieme ai poemetti di Giacomino da Verona, alla Vita in versi di Santa Margherita, quella di Santa Caterina, e altri testi minori, in un ambiente vicino, se non interno, al francescanesimo settentrionale. Tutto ciò vale in particolar modo per il codice trecentesco Marciano it. Z 13 (4744) - l'altro testimone è il codice di Siviglia, Colombino 7-1-52. Quanto al codice Marciano, su basi per lo più iconografiche, è stata avanzata l'ipotesi d'una committenza femminile, con argomentazioni che qui tralasciamo (in favore d'un interesse d'ordine testuale), riguardanti Pavia e qualche donna vicina a confraternite e ordini minori. ${ }^{18}$ Verlato, valutando l'ipotesi, sottolinea da una parte, in linea con tutti gli studi filologici, l'assetto linguistico veronese di molti testi - il che renderebbe più complessa, ma non impossibile, una totale realizzazione a Pavia - e d'altra parte, in prospettiva codicologica, evidenzia la difficoltà di trattare il manoscritto in intero, giacché si tratta d'un composito, per quanto $a b$ antiquo, che dunque lascerebbe adito a stratificazioni, anche d'ordine materiale. ${ }^{19}$ Trasponendo l'ipotesi femminile, che riguarda soprattutto le preghiere relate in quei codici, dal piano iconografico a quello linguistico, Verlato espone i vari elementi, pro e contro l'origine femmi-

15 Monumenti antichi di dialetti italiani; si fa qui riferimento ai testi che, tratti dal codice Marciano it. Z 13 (4744), Mussafia pone nella “sezione G” della sua edizione (pp. 86-101).

16 Verlato, Note filologiche.

17 Sermoni e preghiere in versi in antico veronese. 1. Dell'amore di Gesù; Sermoni e preghiere in versi in antico veronese. 2. Lodi della Vergine.

18 Si veda Miniature a Brera, pp. 130-145.

19 Verlato, Note filologiche, p. 84. 
nile dei testi. Le occorrenze di un io orante femminile sono abbondanti. Eccone solo alcune: ${ }^{20}$

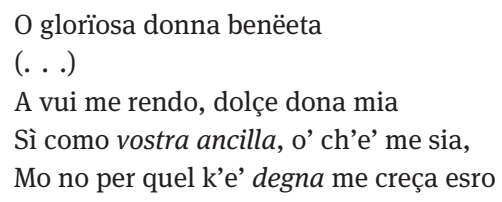

Tanto son rea e povra e nua de fè

Meser Jesù Cristo, Re de li altri re

K'el de mi tapina avrà merçé

(vv. 41-42)

Verlato fa giustizia inoltre dell'ipotesi, da altri avanzata, che questi femminili possano riferirsi non tanto a una donna orante ma genericamente all'anima di chi prega, giacché rileva il paradosso per cui, stante la prosopopea dell'anima, si dovrebbe ammettere che l'anima stessa abbia un'anima, allorché si leggono versi come questo: ${ }^{21}$

\section{E l'anema mia recivi en pax}

Êl santo paraïso, s'el te plas

(vv. 183-184)

Allo stesso modo, con Verlato e Zvonareva, bisogna rilevare due occorrenze contraddittorie, in cui, nella concordia dei testimoni manoscritti, l'io orante parrebbe assumere d'improvviso una fisionomia maschile.

Mo ben confesso, contrito e compunto,

Tu me revella l'ora e 'l dì e 'l ponto

(vv. 277-278)

E in una seconda preghiera, che cambia il metro - dal distico di endecasillabi alla quartina di alessandrini - l'orante è definito, al maschile, peccaor (v. 399).

Quanto alla prima occorrenza - «ben confesso, contrito e compunto»-, credo si possa ammettere l'ipotesi d'un sintagma formulare, o meglio rituale che prescinda così dal genere dei locutori -, e come tale assunto nel testo poe-

20 Leggiamo da Sermoni e preghiere in versi in antico veronese. 2. Lodi della Vergine.

21 Verlato, Note filologiche, p. 81. 
tico. Espressioni affini si trovano stabili negli articoli teologici: peccator vere contritus et confessus; confessus et contritus è inoltre formula ufficiale di remissione e d'indulgenza, variamente attestata, talvolta con l'inserzione d'altri aggettivi - bene confessus et dolens, ad esempio. ${ }^{22}$ La troviamo inoltre, per di più in alternanza di genere grammaticale (femminile/maschile), in una preghiera veneta, trecentesca, edita da Flaminio Pellegrini: «Qualunchena persona (. . .), confeso e contrito de li so peccadi». ${ }^{23}$ Quanto all'altro luogo, il maschile peccaor - sono peccatore e «al cor n’ò gran temança», dice il verso -, è da valutare l'ipotesi di un maschile d'estensione indeterminata - come, in certa misura, i vari «ogn'om» che appiano nel testo delle nostre preghiere. E si può inoltre addurre un caso, veneto, ancora nelle preghiere edite da Pellegrini, che parrebbe utilizzare la morfologia maschile, "peccatore", in un sintagma altrimenti femminile: "per mi peccatore toa serviciale». ${ }^{24}$ Verlato, seguito da Zvonareva, segnala tuttavia un caso più complesso d'apparente contraddizione di genere; più complesso perché in rima e dunque prescrittivo. Si tratta dei versi 289-290.

Ke tu cun tuto lor, Pare divin,

Abe merçé de mi, lasso tapin

Così leggiamo nell'edizione di Zvonareva e già prima in quella di Mussafia. L'apparato tuttavia dimostra come ambedue i testimoni abbiano il femminile: «lassa tapina» il codice Marciano, «lassa topina» quello di Siviglia. Il maschile «lasso tapin» risulta quindi da un emendamento. Questo maschile in rima, secondo Verlato, sarebbe obbligatorio - considerato il verso che precede - e metterebbe in dubbio l'origine femminile del testo, lasciando pensare piuttosto a un orante maschile, riadattato in seguito lungo la tradizione - stante le altre occorrenze - alla voce di una donna. ${ }^{25}$ La correzione - che quindi, oltre ad emendare, ricade sull'ordine "comunicativo", riassegnando l'enunciazione del testo da una donna ad un uomo - pare onerosa, tenuto conto soprattutto d'altre occorrenze in rima relative all'io orante, altrettanto prescrittive dunque e che impongono il femminile.

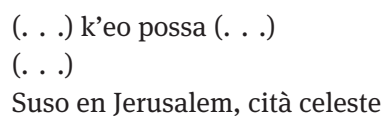

22 La formula appare fra l'altro nel cosiddetto "Perdono d'Assisi" (1310) - in ambito francescano dunque, da dove provengono, verosimilmente, anche le preghiere in questione: «quicunque venerit et intraverit praedictam ecclesiam, bene confessus et contritus, sit absolutus a culpa».

23 Documenti inediti in dialetto veneto, p. 155.

24 Ibid.

25 Verlato, Note filologiche, p. 83. 
Cun nova stola e cun nove veste

Esro recevua per citaïna

Da lo Fiiol de la Vergen raïna.

(vv. 339-344)

Eo, ancilla misera e cativa,

Cun gran merçé te prego en ogna guisa

(vv. 353-354)

E no guardar, Segnor, a mi lassa cativa, Mo de çò te recordo ke tu portassi morto

Per livrar-me de pena e da la morto eniga

(vv. 417-419)

Qualora non si voglia mettere a testo l'assonanza fra tronca e piana, ${ }^{26}$ presente concordemente in ambedue i codici, divin : tapina, il rispetto dell'io emittente, coi vari obblighi verso il genere femminile appena documentati, potrebbe allora, tutt'al più, limitare l'intervento all'apocope di tapina, facendo rimare Pare divin : lassa tapin. Per quanto a rigore imprevista, «arcaica e marginale $»^{27}$ anche in quelle aree venete più caratterizzate dall'apocope ${ }^{28}-$, la caduta della $-a$ finale è tuttavia attestata, e accolta dagli editori, in altri luoghi delle stesse preghiere: abbiamo infatti «per quella glorios Vergen benegna» (v. 158), così trasmesso, stando all'apparato, da ambedue i codici, mentre altrove leggiamo «e tanto me par ella dur novella» (Del Giudizio Universale, v. 37). In generale nella poesia delle origini si trovano altre occorrenze del troncamento in $-a$ : «esta matin», in rima nell'Anonimo Genovese. ${ }^{29}$

I casi di contraddizione fra i generi non sembrano in sintesi tali da cancellare la legittima percezione d'una voce orante al femminile - non già di un'origine autoriale, perché ovviamente nulla esclude che un uomo abbia scritto le preghiere per la recitazione d'una religiosa o d'una comunità femminile; insegna fra gli altri il caso d'Abelardo e degli inni per il Paracleto: «ad festas choreas celibes / ex more venite virgines». Ricollocare una presenza femminile in quei testi è utile comunque alla comparazione - dal Veneto alla Toscana.

Le preghiere veronesi, nel testimone Marciano, sono parte di un più vasto libro devoto, in latino e in volgare, di pietà e d'agiografia, centrato in preva-

26 In Monumenti antichi di dialetti italiani, p. 22, Mussafia notava la mancanza di quest'omofonia nei testi da lui pubblicati, ma a solo patto di correggerne alcune.

27 Serianni, La lingua poetica italiana, p. 124.

28 Testi veronesi dell'età scaligera, pp. 116-137, ma si vedano le osservazioni di Stussi in Testi veneziani del Duecento e dei primi del Trecento, pp. XXXIV-XXXV.

29 Anonimo Genovese, Rime e ritmi latini, p. 313. 
lenza su figure e modelli femminili: la Vergine, Santa Margherita e Santa Caterina. Pertanto, oltre il plausibile, a nostro avviso, orante femminile, nel codice Marciano è lo stesso modello di santità a porre la donna al centro del testo. Quest'ovvia constatazione serve da ponte verso analoghe strutture librarie circolanti in Toscana negli stessi anni, ovvero nei primi due decenni del Trecento. Affinità tematiche possono scorgersi in effetti con un codice piuttosto noto della Toscana religiosa e in certa misura laudistica, il Riccardiano 1738, datato nel colophon al 1317. Il manoscritto, interamente volgare, tramanda un Sermone sull'anima, una Questione de' vizi, la redazione toscana dell'inno Rayna possentissima, una Vita della Vergine e un Pianto della Vergine, in prosa, la Vita in prosa di Santa Margherita, quella in decima rima di Santa Caterina ad opera di Garzo, un'Esposizione del Padre Nostro, una preghiera alla Vergine, uno stralcio del Vangelo di Giovanni, e nelle carte che seguono il colophon qualcuno ha trascritto la lauda Voi c'avete fame de l'amore. Le tangenze col codice Marciano, lombardo-veneto, non sono insignificanti: in ambedue i manoscritti, il settentrionale e il toscano, le sante oggetto di narrazione sono Margherita e Caterina; sia il Marciano che il Riccardiano tramandano una parafrasi commentata del Padre Nostro; ambedue riportano il medesimo estratto, l'inizio, del Vangelo di Giovanni (I, 1-14), e in entrambi i codici compaiono intercalate varie forme di preghiere alla Vergine. Nulla - sia chiaro -, a differenza di quanto avviene col manoscritto veneto, indica nel Riccardiano un'esplicita fruizione femminile; si può solo notare la prevalenza dei soggetti femminili e, per quel che valgono, le affinità redazionali col manoscritto di Venezia. Dal punto di vista formale e stilistico, andrà notata invece la consistenza nel libro toscano dei testi in prosa, minoritari nel codice veneto. Sembra questa nella comparazione dei due libri una specificità del caso toscano, già aperto ai primi del secolo XIV al volgarizzamento agiografico, al sermone scritto, alla minima questione teologico-morale.

Quest'osservazione, attinente a una "geografia delle forme letterarie”, ricade anche sul nostro tema, ovvero la testualità delle donne ai primi del secolo XIV. Scendendo le latitudini dell'Italia primo-trecentesca, giunti in Toscana, è in effetti più semplice trovare qualche parvenza femminile, l'ombra di una lettrice o d'una scrivente, a ridosso della pietas in prosa, del volgarizzamento devoto, più che intorno alla trasmissione poetica, per quanto anch'essa religiosa. Un caso minimo ma istruttivo viene dal codice fiorentino, Laurenziano Gaddi 187. Bertelli lo data alla fine del Duecento, ritenendone «molto arcaico» l'assetto grafico; ${ }^{30}$ il codice tramanda due prose: il testo italiano delle Meditationes vitae Christi e un Pianto della Vergine; libro di pietà volgare, di devozione pri-

30 Bertelli, I manoscritti della letteratura italiana delle origini (BML), pp. 115-116. 
vata, rivendicato sui primi del Trecento da tale Lisa di Lotto Gherardini. ${ }^{31}$ Alla carta 75r si legge: «explicit liber de Meditationibus Christi, qui est de domina Lisa filia olim Lotti de Gerardinis. Qui scripsit scribat. Scribat semper, cum Domino vivat. Vivat in celis, semper cum Domino felix. Deo gratias amen». Mentre alla carta 85r: «iste liber est domina Lisa filia olim Lotti de Gerardinis, quia ego Lisa dedi dicto domino Baldo Angeli de Aretio chanonicus aretinus qui est amor amor meo».

Lisa, lettrice e proprietaria del libro, può identificarsi con una donna del casato fiorentino dei Gherardini, noto alle cronache due-trecentesche (già in quella del Compagni), d'estrazione feudale, fondiaria e cavalleresca, e per questa via talvolta "ghibellino", condannato in alcuni esponenti nel 1302, e al cui interno si contano diversi personaggi chiamati Lotto (da Lotteringo o Pegolotto), come il padre della Lisa che possedette il libro di devozione volgare. La verosimiglianza di quest'ipotesi permette dunque di collocare le Meditazioni in volgare ${ }^{32}$ e il Pianto della Vergine in mano a una donna dell'aristocrazia fiorentina, e urbana e incastellata nel contado. Da qui discende la possibilità di ricercare i frammenti d'alcuni usi testuali di una donna a contatto d'una pur semplice tradizione letteraria. Conta soprattutto che i due testi del codice gaddiano, Meditazioni e Pianto, siano concepiti come esplicita letteratura femminile, tramite frequenti appelli a un pubblico di "sorelle", "lettrici" e "uditrici"; siamo dunque in presenza di un libro per donne, sicuramente fruito da una donna nel caso del manoscritto gaddiano. Per diversi motivi è difficile dire se anche altre scritture che si ritrovano alle fine del codice siano riconducibili al possesso di Lisa de' Gherardini: e per alcune variazioni grafiche e per la stessa nota che Lisa appone, denunciandosi con l'ego, nella quale sembra dire d'aver dato quel libro, a lei particolarmente caro - parrebbe -, a un uomo, Baldo di Angelo canonico aretino, qui est amor amor meo. Va detto tuttavia che nella carta che precede e in quella che segue la nota di possesso si leggono due semplici e basilari componimenti poetici, di mano comunque trecentesca, e d'ispirazione religiosa e morale. Ambedue i testi $^{33}$ si giocano sulla prosopopea - femminile - della Carità e dell’Umiltà. Ec-

31 Si segnalava l'esistenza di Lisa e del suo libro in Mainini, Gli anni della tradizione, p. 217.

32 Si tratta di un'opera, dalla vasta e importante circolazione, ancora irrisolta dal punto di vista critico-testuale; per un recente tentativo d'organizzarne la tradizione, fra latino e volgare, e di collocarne il senso in un appropriato contesto storico (la cultura dei francescani in Toscana ai confini fra Due e Trecento), si veda Falvay, Tóth, L'autore e la trasmissione.

33 Che devono aver fatto parte d'un ciclo didascalico sui vizi e le virtù, giacché li ritroviamo messi "in contesto" fra le carte d'altri manoscritti tre-quattrocenteschi (ad esempio il laurenziano Plut. 90 inf. 47, 41vr.). 
cone - per avere un saggio del tenore linguistico e culturale - un estratto, in diplomatica, dal primo componimento (84v).

\section{Charità}

Tanto gli piaccio $\cdot$ io karita a dio $\cdot$ et siglio gratioso il mio et fecto $\cdot$ Quantuque ongni piacere gli sia - diletto $\cdot$ Et non gliene veruna mai quantio $\cdot$ Che cio vero et proprio il dicer mio, E cho i santi et savi channo detto . Chel suo figliuolo Christo benedetto venne per me venne per me nel mondo $\cdot$ et po morio (. . .).

Al netto delle mancanze o delle imperfezioni, la forma di questi componimenti è riconducibile al sonetto. La doppia prosopopea femminile, all'interno d'un codice già fortemente marcato in senso femminile, per testi e possessori, continua a mantenerci in un'atmosfera compatibile con la cultura, le letture e gli interessi di una donna. Però certo i due componimenti non potranno essere valutati come testimonianze né di una poesia né di un volgare femminile. A questo scopo piuttosto si potrebbe dire qualcosa (poco) sulla lingua di Lisa de' Gherardini, a partire dalla nota di possesso (quella sì plausibilmente autografa). Il suo latino è un grafico rivestimento del volgare, che, emulando le note di possesso librarie e clericali, infrange in realtà la flessione dei casi, sino a quel meo che abolisce la declinazione. Altrove (75r) - ma di una mano apparentemente diversa - si legge un dico ad apertura d'una frase avventizia in latino - «dico quod nolo». Il verbo è scritto due volte; la prima dicho, poi scartato in favore della forma dico - il che potrebbe dimostrare una titubanza nella grafia della velare, fra una resa scritta alla volgare e quella latina.

In ogni caso per simili ricerche, ovvero per tentare di far emergere un volgare femminile nei dislivelli della trasmissione latina, si possono utilizzare ben altre fonti: ad esempio, in certa misura, il Memoriale di Angela da Foligno. Qui gli studi ${ }^{34}$ hanno già messo in luce vari elementi - però nella maggior parte dei casi riconducibili al sermo humilis in latino del frate scrittore, dotato apparentemente d'una sua stilistica, pur nella struttura in realtà romanza. Alla voce di Angela da Foligno potranno al massimo, e per ipotesi, imputarsi i volgarismi di lessico o certe pieghe sintattiche: ad esempio, dietro un «Domine, capias pietatem de me», ${ }^{35}$ non canonico nel latino delle suppliche (che potrebbe avere piuttosto un miserere mei), si può immaginare vi fosse, nell'eventuale voce di Angela, un «ti prenda pietà di me», giro verbale frequente nella scrittura volgare e nel linguaggio laudistico: «di nui Ti prenda pietade» nel Laudario Cortonese. ${ }^{36}$ Analoghi esercizi si possono fare con altri testi latini,

34 Si veda fra gli altri Donnini, Appunti sulla lingua.

35 Angela da Foligno, Memoriale, p. 27.

36 Laudario di Cortona, p. 132. 
non molti, due-trecenteschi che suppongono all'origine un'enunciazione femminile; coi Sermones d'Umiltà da Faenza per esempio. Nonostante siano relati da manoscritti decisamente tardi, ${ }^{37}$ i testi latini d'Umiltà consegnano volgarismi di vario tipo, tracce della lingua che sottostà alla loro concezione: domicella, zuncata, foleolina, quali esempi di lessico e morfologia.

Per concludere; i dislivelli - quelli della trasmissione manoscritta, delle forme grafiche e linguistiche - al cui interno può ipotizzarsi una presenza femminile, nei secoli d'origine della letteratura italiana, permettono di riconoscere la lingua delle donne solo per lacerti. Possono far velo inoltre a questa ricerca fenomeni di autocensura; ovvero non è da escludere che in certi casi le donne silenzino, allo scritto, la loro specificità linguistica. In un volgarizzamento primo-trecentesco della Regola di San Benedetto, appartenuto - «fatto fare», dice la rubrica del Riccardiano 2858 - alle monache fiorentine di San Pier Maggiore, ${ }^{38}$ il testo è assunto al maschile, laddove nel testimone latino della Regula appartenuto alle stesse monache sui margini del libro si legge costantemente la sostituzione di frater in soror e di abbas in abbatissa. Sembra in sintesi che in epoca alta la presenza femminile sopravviva nell'occasionalità delle testimonianze superstiti, la cui "leggibilità" dipende inoltre dalla dialettica - non per forza consapevole - tra la norma ereditata, la sua riproduzione, e la possibilità per la donna di non scomparire, di sussistere almeno in parte (in serie di rimanti, in glosse e note a margine, in volgarismi orali affioranti nello scritto) come soggetto d'un discorso.

37 Umiltà da Faenza, Sermones.

38 Che del resto negli anni in cui si realizzò il codice compaiono nei documenti quali donne «instructe licterate», capaci - parrebbe - di dare insegnamento «in legendo et cantando» (Firenze, Archivio di Stato, Diplomatico, S. Pier Maggiore, 20 settembre 1307). 


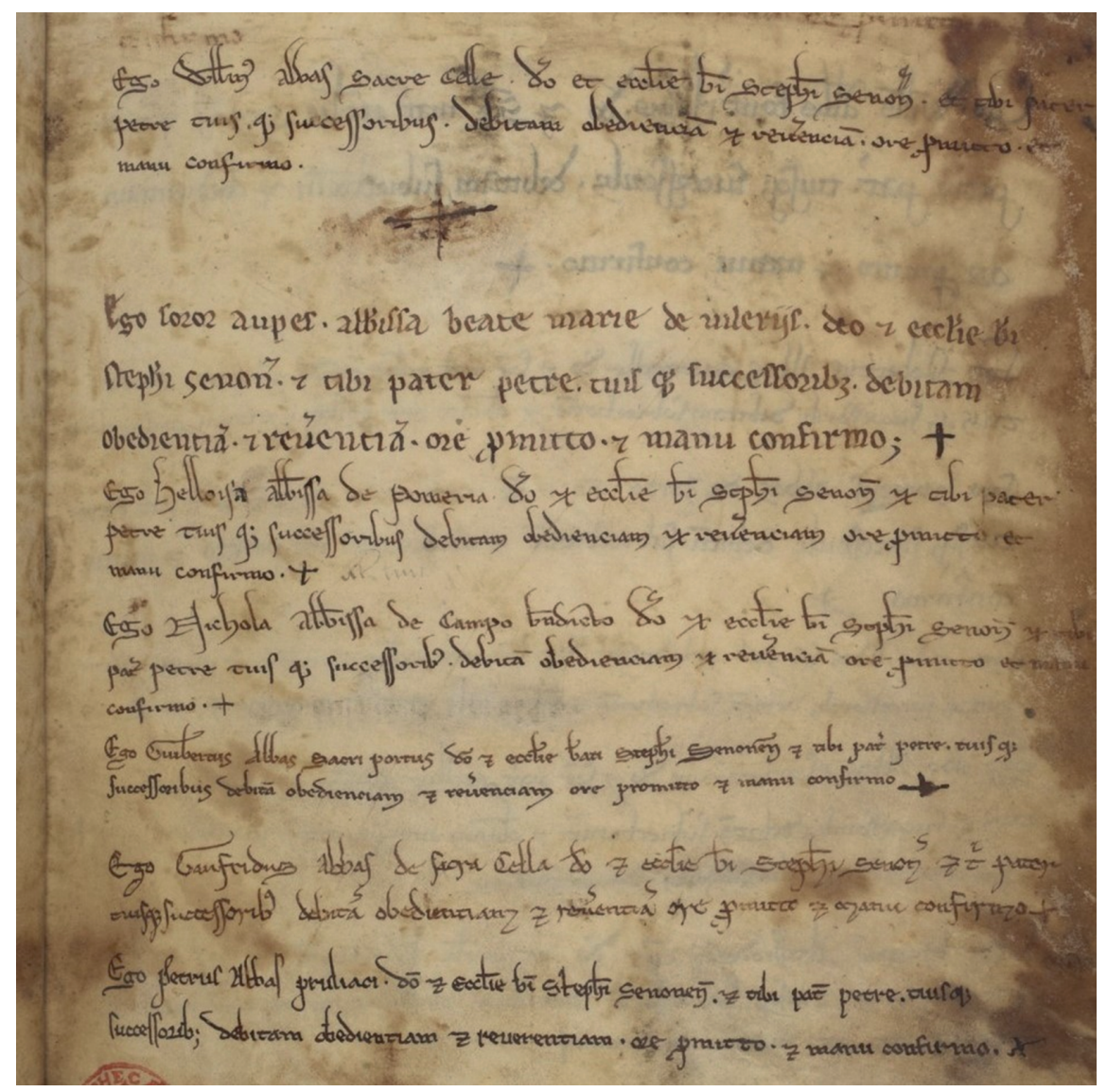

Figura 11.1: Paris, BNF, lat. 934, Pontificale di Sens. 